\title{
Gradient systems with sublinear term near the origin and asymptotically linear term near infinity
}

Hongrui Cai and Jiabao Su

"Correspondence: eulen26@gmail.com School of Mathematical Sciences, Capital Normal University, Beijing, 100048, People's Republic of China

\begin{abstract}
In this paper we study the existence of nontrivial solutions for a sublinear gradient system with a nontrivial critical group at infinity.

MSC: $35 \mathrm{~J} 10 ; 35 \mathrm{~J} 65 ; 58 \mathrm{E} 05$
\end{abstract}

Keywords: gradient system; sublinear; critical group; Morse theory

\section{Introduction}

In this paper, we are concerned with the following gradient system

$$
\begin{cases}-\Delta u=F_{u}(x, u, v), & x \in \Omega, \\ -\Delta v=F_{v}(x, u, v), & x \in \Omega, \\ u=v=0, & x \in \partial \Omega,\end{cases}
$$

where $\Omega \subset \mathbb{R}^{N}$ is a bounded open domain with a smooth boundary $\partial \Omega$ and $F_{u}$ designates the partial derivative with respect to $u$ of the nonlinearity $F: \Omega \times \mathbb{R}^{2} \rightarrow \mathbb{R}$. The solutions of such systems are steady-states of reaction-diffusion systems arising in many applied sciences such as biology, chemistry, ecology or physics. It is well known that (GS) has variational structure when the nonlinearity $F$ satisfies the subcritical growth condition

(F) $F \in C^{1}\left(\Omega \times \mathbb{R}^{2}, \mathbb{R}\right)$ and there are $C>0$ and $2<p<2^{*}$ such that

$$
|\nabla F(x, z)| \leq C\left(1+|z|^{p-1}\right), \quad \text { for } x \in \Omega, z=(u, v) \in \mathbb{R}^{2},
$$

where $2^{*}=\frac{2 N}{N-2}$ if $N \geq 3$ and $2^{*}=\infty$ if $N=1,2$.

That is, the solutions of (GS) can be found as critical points of the following functional

$$
\Phi(u, v):=\frac{1}{2} \int_{\Omega}|\nabla u|^{2}+|\nabla v|^{2} d x-\int_{\Omega} F(x, u, v) d x
$$

defined on $E:=H_{0}^{1}(\Omega) \times H_{0}^{1}(\Omega)$ which is a Hilbert space endowed with the inner product

$$
\langle z, w\rangle=\langle(u, v),(\phi, \psi)\rangle=\int_{\Omega}(\nabla u \nabla \phi+\nabla v \nabla \psi) d x, \quad z=(u, v), w=(\phi, \psi) \in E
$$

○2013 Cai and Su; licensee Springer. This is an Open Access article distributed under the terms of the Creative Commons Attribution License (http://creativecommons.org/licenses/by/2.0), which permits unrestricted use, distribution, and reproduction in any medium, provided the original work is properly cited. 
and the associated norm

$$
\|z\|^{2}=\int_{\Omega}|\nabla z|^{2} d x=\int_{\Omega}|\nabla u|^{2}+|\nabla v|^{2} d x, \quad z=(u, v) \in E .
$$

By the compact Sobolev embedding $E \hookrightarrow L^{q}(\Omega) \times L^{q}(\Omega)$ with $q \in\left[1,2^{*}\right)$, under the global assumption $(F)$, the functional $\Phi$ is well defined and is of class $C^{1}$ (see [1]) with its Fréchet derivative

$$
\left\langle\Phi^{\prime}(u, v),(\varphi, \psi)\right\rangle=\int_{\Omega}(\nabla u \nabla \varphi+\nabla v \nabla \psi) d x-\int_{\Omega}(\nabla F(x, u, v),(\varphi, \psi)) d x
$$

for $(u, v),(\varphi, \psi) \in E$. The weak solutions to (GS) in $E$ are exactly critical points of $\Phi$ in $E$.

We make some conventions. We use $|\cdot|$ and $(\cdot, \cdot)$ to denote the norm and the inner product in $\mathbb{R}^{2}$ and use $z=(u, v)$ to denote an element in $\mathbb{R}^{2}$ and $E$. Bz denotes the matrix product in $\mathbb{R}^{2}$ for a $2 \times 2$ matrix $B$ and $z=(u, v) \in \mathbb{R}^{2}$. We use 0 to denote the origin in various spaces. Let $\mathcal{M}_{2}(\Omega)$ be the set of all continuous, cooperative and symmetric matrix functions on $\mathbb{R}^{2}$. A matrix function $A \in \mathcal{M}_{2}(\Omega)$ takes the form

$$
A(x)=\left(\begin{array}{ll}
a(x) & b(x) \\
b(x) & c(x)
\end{array}\right)
$$

with the functions $a, b, c \in C(\bar{\Omega}, \mathbb{R})$ satisfying the conditions that $b(x) \geq 0$ for all $x \in \bar{\Omega}$, which means $A$ is cooperative, and that $\max _{x \in \bar{\Omega}}\{a, c\}>0$.

When $F$ satisfies $\nabla F(x, 0)=0, F(x, 0)=0$ for $x \in \Omega$, the system (GS) admits a trivial solution $z=0$. We are interested in the nontrivial solutions for (GS). In the current paper we apply the Morse theory to study the existence of nontrivial solutions of (GS) when the problem is sublinear near the origin and is asymptotically linear near infinity.

We make the following assumption near the origin.

$\left(F_{0}\right) \nabla F(x, 0)=0, F(x, 0)=0$ and there are $\delta>0$ and $1<\sigma<2$ such that

$$
0<(\nabla F(x, z), z) \leq \sigma F(x, z) \text { for } z \in \mathbb{R}^{2} \text { with } 0<|z| \leq \delta, x \in \Omega
$$

In order to state the assumptions on the nonlinearity at infinity, we need some basic facts about the eigenvalue problem of linear gradient system. For a given matrix $A \in \mathcal{M}_{2}(\Omega)$, it is known (see $[2,3])$ that the corresponding linear system

$$
\begin{cases}-\Delta z=\lambda A(x) z, & x \in \Omega \\ z=0, & x \in \partial \Omega\end{cases}
$$

admits a sequence of distinct eigenvalues of finite multiplicity

$$
0<\lambda_{1}(A)<\lambda_{2}(A)<\cdots<\lambda_{k}(A)<\cdots
$$

such that $\lambda_{k}(A) \rightarrow \infty$ as $k \rightarrow \infty$. According to $A$, the space $E$ can be split as

$$
E=E_{A}^{-} \oplus E_{A} \oplus E_{A}^{+},
$$


where

$$
E_{A}=\operatorname{ker}(\Delta+A), \quad E_{A}^{-}=\bigoplus_{\lambda_{k}(A)<1} \operatorname{ker}\left(\Delta+\lambda_{k}(A) A\right)
$$

The numbers $m(A)=\operatorname{dim} E_{A}^{-}, n(A)=\operatorname{dim} E_{A}$ are well determined and finite.

We assume that the nonlinear system (GS) is asymptotically linear at infinity in the sense that the function $F$ satisfies

$\left(F_{\infty}\right)$ there is a matrix $B_{\infty} \in \mathcal{M}_{2}(\Omega)$ such that

$$
\nabla F(x, z)-B_{\infty}(x) z=o(|z|), \quad|z| \rightarrow \infty, z \in \mathbb{R}^{2}, x \in \Omega .
$$

Associated to $B_{\infty}$, we set $E_{\infty}^{-}=E_{B_{\infty}}^{-}, E_{\infty}=E_{B_{\infty}}, E_{\infty}^{+}=E_{B_{\infty}}^{+}$. Denote $m_{\infty}=m\left(B_{\infty}\right):=\operatorname{dim} E_{\infty}^{-}$, $n_{\infty}=n_{B_{\infty}}:=\operatorname{dim} E_{\infty}$. We say that the system (GS) is nonresonant at infinity if $n_{\infty}=0$, while it is resonant at infinity if $n_{\infty}>0$.

We first consider the nonresonance case. We have the following.

Theorem 1.1 Assume that $F$ satisfies $\left(F_{0}\right),\left(F_{\infty}\right)$ and $n_{\infty}=0$. Then (GS) has at least one nontrivial weak solution in $E$.

Next we consider the resonance case. We need additional assumptions on $F$ near infinity.

$\left(F_{\infty 1}\right) \lambda_{1}\left(B_{\infty}\right)=1$ and

$$
\lim _{|z| \rightarrow \infty}\left(2 F(x, z)-\left(B_{\infty} z, z\right)\right)=-\infty \quad \text { uniformly in } \Omega
$$

$\left(F_{\infty 2}^{ \pm}\right) \lambda_{k}\left(B_{\infty}\right)=1$ for some $k \geq 2$. For $z_{n}=y_{n}+w_{n}$, where $y_{n} \in E_{\infty}, w_{n} \in E_{\infty}^{\perp},\left\|z_{n}\right\| \rightarrow \infty$ and $\frac{\left\|y_{n}\right\|}{\left\|z_{n}\right\|} \rightarrow 1$ imply that there exist $\delta>0$ and $N \in \mathbb{N}$ such that

$$
\pm \int_{\Omega}\left(\nabla F\left(x, z_{n}\right)-B_{\infty} z_{n}, y_{n}\right) d x \geq \delta \quad \text { for } n \geq N
$$

Theorem 1.2 Let $F$ satisfy $\left(F_{0}\right),\left(F_{\infty}\right)$ and $\left(F_{\infty 1}\right)$. Then (GS) has at least one nontrivial weak solution in $E$. Moreover, if $F$ is even in $z$, then (GS) has infinitely many nontrivial weak solutions in $E$.

Theorem 1.3 Let $F$ satisfy $\left(F_{0}\right),\left(F_{\infty}\right)$ and $\left(F_{\infty 2}^{ \pm}\right)$. Then (GS) has at least one nontrivial weak solution in $E$.

Now we give some remarks and comments. The gradient system represents the steadystate case of reaction-diffusion system which is a model for problems arising from biology, chemistry, physics and ecology, etc. In this paper we look for nontrivial solutions for the system (GS) via Morse theory. When the problem is resonant at infinity, we impose on the nonlinearity $F$ the global assumption $\left(F_{\infty}^{ \pm}\right)$to ensure the compactness and clear description of critical groups for $\Phi$ at infinity. $\left(F_{\infty}^{ \pm}\right)$can be regarded as a variant of the famous 
Landesman-Lazer type resonance condition [4] which can be formulated as

$$
\left\{\begin{array}{l}
\left|\nabla F(x, z)-B_{\infty}(x) z\right| \leq C, \quad x \in \Omega, z \in \mathbb{R}^{2}, \\
\lim _{\|y\| \rightarrow \infty} \int_{\Omega}\left(2 F(x, y)-\left(B_{\infty}(x) y, y\right)\right) d x= \pm \infty, \quad y \in E_{\infty} .
\end{array}\right.
$$

See [5] for details. Near the origin we impose $\left(F_{0}\right)$, which means that $\nabla F$ is sublinear or $F$ is sub-quadratic near zero. This kind of condition caught our attention first in a preprint by Liu and Wu [6] where a single elliptic equation was considered. This is the first use for gradient system in the current paper.

The asymptotically linear gradient systems (GS) have received some attention for years. We mention some recent related works [7-12] and the references therein. In these works, existence and multiplicity of nontrivial solutions for (GS) were obtained by combining various arguments involving Morse theory, saddle point reduction method (see [9-11]) and three critical point theorem (see [13]), etc. All above mentioned works dealt with the case that at least one of the critical groups of $\Phi$ at 0 is nontrivial somewhere. In the present paper, we study via Morse theory the case that all critical groups of $\Phi$ at 0 are trivial under the condition $\left(F_{0}\right)$. Due to $\left(F_{0}\right)$, the saddle point reduction methods [9-11] cannot be applied and there is no linking at 0 . Comparing with known ones, the existence and multiplicity results for (GS) are all new. See more remarks in the last section of the paper.

The paper is organized as follows. In Section 2, we collect some basic abstract tools. In Section 3 we compute the critical groups at zero and infinity. The proofs of Theorems 1.11.3 and comments are given in Section 4.

\section{Preliminary}

In this section we cite some preliminaries that will be used to prove the main results of the paper. We first collect some results on Morse theory (see $[14,15])$ for a $C^{1}$ functional $\Phi$ defined on a Hilbert space $E$.

Let $\Phi \in C^{1}(E, \mathbb{R})$. Denote for $c \in \mathbb{R}$

$$
\Phi^{c}=\{z \in E: \Phi(z) \leq c\}, \quad \mathcal{K}_{c}=\left\{z \in E: \Phi^{\prime}(z)=0, \Phi(z)=c\right\}
$$

We say that $\Phi$ possesses the deformation property at the level $c \in \mathbb{R}$ if for any $\bar{\epsilon}>0$ and any neighborhood $\mathcal{N}$ of $\mathcal{K}_{c}$, there are $\epsilon>0$ and a continuous deformation $\eta: E \times[0,1] \rightarrow E$ such that

(1) $\eta(z, t)=z$ for either $t=0$ or $z \notin \Phi^{-1}[c-\bar{\epsilon}, c+\bar{\epsilon}]$;

(2) $\Phi(\eta(z, t))$ is non-increasing in $t$ for any $z \in E$;

(3) $\eta\left(\Phi^{c+\epsilon} \backslash \mathcal{N}\right) \subset \Phi^{c-\epsilon}$.

We say that $\Phi$ possesses the deformation property if $\Phi$ possesses the deformation property at each level $c \in \mathbb{R}$.

In applications the deformation property is ensured by the Palais-Smale condition or the Cerami condition.

We say that $\Phi$ satisfies the Palais-Smale condition at the level $c \in \mathbb{R}$ if any sequence $\left\{z_{n}\right\} \subset E$ satisfying $\Phi\left(z_{n}\right) \rightarrow c$ and $\Phi^{\prime}\left(z_{n}\right) \rightarrow 0$ as $n \rightarrow \infty$ has a convergent subsequence. $\Phi$ satisfies the Palais-Smale condition if $\Phi$ satisfies the Palais-Smale condition at each $c \in \mathbb{R}$. We say that $\Phi$ satisfies the Cerami condition $[16,17]$ at the level $c \in \mathbb{R}$ if any sequence $\left\{z_{n}\right\} \subset E$ satisfying that $\Phi\left(z_{n}\right) \rightarrow c,\left(1+\left\|z_{n}\right\|\right)\left\|\Phi^{\prime}\left(z_{n}\right)\right\| \rightarrow 0$ as $n \rightarrow \infty$ has a con- 
vergent subsequence. $\Phi$ satisfies the Cerami condition if $\Phi$ satisfies the Cerami condition at each $c \in \mathbb{R}$.

If $\Phi$ satisfies the Palais-Smale condition or the Cerami condition, then $\Phi$ possesses the deformation property $[14,16]$.

Let $z_{0}$ be an isolated critical point of $\Phi$ with $\Phi\left(z_{0}\right)=c \in \mathbb{R}$, and $U$ be a neighborhood of $z_{0}$. The group

$$
C_{q}\left(\Phi, z_{0}\right):=H_{q}\left(\Phi^{c} \cap U, \Phi^{c} \cap U \backslash\left\{z_{0}\right\}\right), \quad q \in \mathbb{Z}
$$

is called the $q$ th critical group of $\Phi$ at $z_{0}$, where $H_{*}(A, B)$ denotes a singular relative homology group of the pair $(A, B)$ with integer coefficients.

Let $\mathcal{K}=\left\{z \in E: \Phi^{\prime}(z)=0\right\}$. Assume that $\Phi(\mathcal{K})$ is bounded from below by $a \in \mathbb{R}$ and $\Phi$ possesses the deformation property at all $c \leq a$. Then the group

$$
C_{q}(\Phi, \infty):=H_{q}\left(E, \Phi^{a}\right), \quad q \in \mathbb{Z}
$$

is called the $q$ th critical group of $\Phi$ at infinity [18].

Assume that $\Phi$ satisfies the deformation property and $\mathcal{K}$ is a finite set. The Morse type numbers of the pair $\left(E, \Phi^{a}\right)$ are defined by $M_{q}:=\sum_{z \in \mathcal{K}} \operatorname{dim} C_{q}(\Phi, z)$, and the Betti numbers of the pair $\left(E, \Phi^{a}\right)$ are defined by $\beta_{q}:=\operatorname{dim} C_{q}(\Phi, \infty)$.

Proposition 2.1 Assume that $\Phi \in C^{1}(E, \mathbb{R})$ possesses the deformation property, $\# \mathcal{K}<\infty$, and all $M_{q}, \beta_{q}$ are finite and only finitely many of them are nonzero. Then it holds

$$
\begin{aligned}
& \sum_{j=0}^{q}(-1)^{q-j} M_{j} \geq \sum_{j=0}^{q}(-1)^{q-j} \beta_{j} \quad \text { (Morse inequality), } \\
& \sum_{q=0}^{\infty}(-1)^{q} M_{q}=\sum_{q=0}^{\infty}(-1)^{q} \beta_{q} \quad \text { (Morse equality). }
\end{aligned}
$$

If $\mathcal{K}=\emptyset$, then $\beta_{q}=0$ for all $q \in \mathbb{Z}$. From (2.1) one can deduce that $M_{q} \geq \beta_{q}$ for all $q \in \mathbb{Z}$. Thus if $\beta_{q} \neq 0$ for some $q_{*} \in \mathbb{Z}$, then $\Phi$ must have a critical point $z^{*}$ with $C_{q_{*}}\left(\Phi, z^{*}\right) \varsubsetneqq 0$. If $\mathcal{K}=\left\{z^{*}\right\}$, then $C_{q}(\Phi, \infty) \cong C_{q}\left(\Phi, z^{*}\right)$ for all $q \in \mathbb{Z}$. Thus if $C_{q}(\Phi, \infty) \nsucceq C_{q}\left(\Phi, z^{*}\right)$ for some $q \in \mathbb{Z}$, then $\Phi$ must have a new critical point. Therefore the basic idea in applying Morse theory to find critical points of $\Phi$ is to compute critical groups both at infinity and at known critical points clearly and then to find unknown critical points by applying formulas (2.1) and (2.2).

Now we state an abstract result for the critical groups at infinity.

Proposition 2.2 Let the functional $\Phi: E \rightarrow \mathbb{R}$ take the form

$$
\Phi(z)=\frac{1}{2}\langle\mathcal{L} z, z\rangle+\Psi(z)
$$

where $\mathcal{L}: E \rightarrow E$ is a self-adjoint linear operator such that 0 is isolated in $\sigma(\mathcal{L})$, the spectrum of $\mathcal{L}$. Assume that $\Psi \in C^{1}(E, \mathbb{R})$ satisfies

$$
\left\|\Psi^{\prime}(z)\right\|=o(\|z\|) \quad \text { as }\|z\| \rightarrow \infty .
$$


Denote $V:=\operatorname{ker} \mathcal{L}, W:=V^{\perp}=W^{+} \oplus W^{-}$, where $W^{ \pm}$are subspaces on which $\mathcal{L}$ is positive (negative) definite. Assume that $\mu=\operatorname{dim} W^{-}$and $v=\operatorname{dim} V$ are finite, and $\Phi$ possesses the deformation property.

(1) If $v=0$, then

$$
C_{q}(\Phi, \infty) \cong \delta_{q, \mu} \mathbb{Z}, \quad q \in \mathbb{Z}
$$

(2) If $v>0$, then

$$
C_{q}(\Phi, \infty) \cong \delta_{q, k^{ \pm}} \mathbb{Z}, \quad k^{+}=\mu, k^{-}=\mu+v
$$

provided $\Phi$ satisfies the angle conditions with respect to $E=V \oplus W$ :

$\left(\mathcal{A C}_{\infty}^{ \pm}\right)$there exist $M>0$ and $\epsilon \in(0,1)$ such that

$$
\pm\left\langle\Phi^{\prime}(z), y\right\rangle \geq 0, \quad \text { for } z=y+w,\|z\| \geq M,\|w\| \leq \epsilon\|z\|, y \in V, w \in W
$$

Proposition 2.1(1) was obtained in [19] (see Remark 5.2 in [14]). Proposition 2.1(2) is a revision of Proposition 3.10 in [18] which was made first in [20] and was remade in [21].

Next we recall an abstract critical point theorem built by Wang in [22].

Proposition 2.3 ([22]) Let $\Phi \in C^{1}(X, \mathbb{R})$, where $X$ is a Banach space. Assume that $\Phi$ possesses the deformation property, is even and bounded from below, and $\Phi(0)=0$. If for any $k \in \mathbb{N}$, there exist $k$-dimensional subspaces $X_{k}$ and $\rho_{k}>0$ such that

$$
\sup _{z \in X_{k} \cap S_{\rho_{k}}} \Phi(z)<0
$$

where $S_{\rho}=\{u \in X \mid\|u\|=\rho\}$, then $\Phi$ has a sequence of critical values $c_{k}<0$ satisfying $c_{k} \rightarrow 0$ as $k \rightarrow \infty$.

Finally, we mention the eigenvalues of the linear gradient system $\left(\mathrm{L}_{\mathrm{A}}\right)$. By the compact embedding $E \hookrightarrow L^{2}(\Omega) \times L^{2}(\Omega)$, for a given $A \in \mathcal{M}_{2}(\Omega)$, there is a compact self-adjoint operator $T_{A}: E \rightarrow E$ associated with $A$ such that

$$
\left\langle T_{A} z, w\right\rangle=\int_{\Omega}(A(x) z, w) d x, \quad z, w \in E .
$$

The operator $T_{A}$ possesses the property that $\lambda(A)$ is an eigenvalue of $\left(\mathrm{L}_{\mathrm{A}}\right)$ if and only if there is nonzero $z \in E$ such that

$$
\lambda(A) T_{A} z=z
$$

$\left(\mathrm{L}_{\mathrm{A}}\right)$ has a sequence of distinct eigenvalues

$$
0<\lambda_{1}(A)<\lambda_{2}(A)<\cdots<\lambda_{k}(A)<\cdots \rightarrow \infty,
$$

and each eigenvalue $\lambda(A)$ of $\left(\mathrm{L}_{\mathrm{A}}\right)$ has a finite multiplicity. All eigenvectors of $\left(\mathrm{L}_{\mathrm{A}}\right)$ form a Hilbertian basis of $E$ and that $E$ can be split as $E=E_{A}^{-} \oplus E_{A} \oplus E_{A}^{+}$, where $E_{A}^{-}, E_{A}^{+}, E_{A}$ are the 
negative, positive definite invariant subspaces and the kernel of $I-T_{A}$, respectively. We refer to $[2,3]$ for more properties related to the eigenvalue problem $\left(\mathrm{L}_{\mathrm{A}}\right)$ and the operator $T_{A}$.

\section{Critical groups and compactness}

In this section we verify the compactness of the functional $\Phi$ and compute the critical groups of $\Phi$ at both zero and infinity. Without loss of generality, we assume that (GS) has finitely many weak solutions so that the trivial solution $z=0$ is an isolated critical point of $\Phi$. We first compute the critical groups $C_{q}(\Phi, 0)$. The idea was from an unpublished preprint by Liu and $\mathrm{Wu}[6]$ where a single elliptic equation was studied.

We work with the functional

$$
\Phi(z)=\frac{1}{2} \int_{\Omega}|\nabla z|^{2} d x-\int_{\Omega} F(x, z) d x, \quad z \in E .
$$

Lemma 3.1 Assume that $F$ satisfies $(F)$ and $\left(F_{0}\right)$, then

$$
C_{q}(\Phi, 0) \cong 0 \quad \text { for all } q \in \mathbb{Z}
$$

Proof Denote $B_{\rho}:=\{z \in E:\|z\| \leq \rho\}$. By definition of critical groups, we can write $C_{q}(\Phi, 0) \triangleq H_{q}\left(B_{\rho} \cap \Phi^{0}, B_{\rho} \cap \Phi^{0} \backslash\{0\}\right)$. We will construct a deformation mapping from $\left(B_{\rho}, B_{\rho} \backslash\{0\}\right)$ to $\left(B_{\rho} \cap \Phi^{0}, B_{\rho} \cap \Phi^{0} \backslash\{0\}\right)$ for $\rho>0$ small.

In the following we use $c_{i}$ to denote positive constants. By $\left(F_{0}\right)$, one deduces that

$$
F(x, z) \geq c_{1}|z|^{\sigma} \quad \text { for } x \in \Omega, z \in \mathbb{R}^{2},|z| \leq \delta .
$$

It follows from $(F)$ and $(2.2)$ that for some $p \in\left(2,2^{*}\right)$,

$$
F(x, z) \geq c_{2}|z|^{\sigma}-c_{3}|z|^{p}, \quad x \in \Omega, z \in \mathbb{R}^{2} .
$$

For $z \in E$ and $s>0$, we have

$$
\begin{aligned}
\Phi(s z) & =\frac{1}{2} s^{2} \int_{\Omega}|\nabla z|^{2} d x-\int_{\Omega} F(x, s z) d x \\
& \leq \frac{1}{2} s^{2}\|z\|^{2}-\int_{\Omega}\left(c_{2}|s z|^{\sigma}-c_{3}|s z|^{p}\right) d x \\
& \leq \frac{1}{2} s^{2}\|z\|^{2}-c_{2} s^{\sigma}\|z\|_{L^{\sigma}}^{\sigma}+c_{3} s^{p}\|z\|_{L^{p}}^{p} .
\end{aligned}
$$

Since $\sigma<2<p$, for each given $z \in E \backslash\{0\}$, there exists $s_{0}=s_{0}(z)>0$ such that

$$
\Phi(s z)<0 \text { for all } 0<s<s_{0} .
$$


Let $z \in E$ be such that $z \neq 0$ and $\Phi(z)=0$. Then

$$
\begin{aligned}
\left.\frac{d}{d s} \Phi(s z)\right|_{s=1} & =\int_{\Omega}|\nabla z|^{2} d x-\int_{\Omega}(\nabla F(x, z), z) d x \\
& =\left(1-\frac{\sigma}{2}\right)\|z\|^{2}-\int_{\Omega}((\nabla F(x, z), z)-\sigma F(x, z)) d x \\
& \geq\left(1-\frac{\sigma}{2}\right)\|z\|^{2}-c_{4} \int_{\Omega}|z|^{p} d x \\
& \geq\left(1-\frac{\sigma}{2}\right)\|z\|^{2}-c_{5}\|z\|^{p}
\end{aligned}
$$

From (3.6), one concludes that there exists $\rho>0$ such that

$$
\left.\frac{d}{d s} \Phi(s z)\right|_{s=1}>0 \quad \text { for } z \in E \text { with } \Phi(z)=0 \text { and } 0<\|z\| \leq \rho
$$

From now on we fix $\rho>0$. We claim that

$$
z \in B_{\rho} \text { and } \Phi(z)<0 \quad \Longrightarrow \quad \Phi(s z)<0 \quad \text { for all } s \in(0,1) \text {. }
$$

Let $z \in B_{\rho}$ and $\Phi(z)<0$. By the continuity of $\Phi$, there exists $\tau \in(0,1]$ such that $\Phi(s z)<0$ for all $s \in(1-\tau, 1)$. We will get (3.8) by proving $\tau=1$. Suppose that $0<\tau<1$. Then there is some $s_{0} \in(0,1-\tau]$ such that

$$
\Phi\left(s_{0} z\right)=0, \quad \Phi(s z)<0 \quad \text { for } s_{0}<s<1
$$

As $s_{0} z \in B_{\rho}$, it follows from (3.7) that

$$
\left.\frac{d}{d s} \Phi\left(s\left(s_{0} z\right)\right)\right|_{s=1}>0
$$

But $\Phi(s z)-\Phi\left(s_{0} z\right)=\Phi(s z)<0$ implies that

$$
\left.\frac{d}{d s} \Phi\left(s\left(s_{0} z\right)\right)\right|_{s=1} \leq 0
$$

This contradicts (3.9). Thus $\tau=1$ and (3.8) holds.

Now define a mapping $\pi: B_{\rho} \rightarrow[0,1]$ as

$$
\pi(z)= \begin{cases}1 & \text { for } z \in B_{\rho} \text { with } \Phi(z) \leq 0 \\ s \in(0,1) & \text { for } z \in B_{\rho} \text { with } \Phi(z)>0 \text { and } \Phi(s z)=0\end{cases}
$$

By (3.5), (3.7) and (3.8), for $z \in B_{\rho}$ with $\Phi(z)>0$, there exists a unique $\pi(z) \in(0,1)$ such that

$$
\begin{cases}\Phi(\pi(z) z)=0, & \\ \Phi(s z)<0 & \text { for all } s \in(0, \pi(z)) \\ \Phi(s z)>0 & \text { for all } s \in(\pi(z), 1)\end{cases}
$$


Thus the mapping $\pi$ is well defined. Moreover, it follows from (3.7), (3.12) and the implicit function theorem that the mapping $\pi$ is continuous in $z$. Define a mapping $\eta:[0,1] \times B_{\rho} \rightarrow$ $B_{\rho}$ by

$$
\eta(s, z)=(1-s) z+s \pi(z) z, \quad s \in[0,1], z \in B_{\rho} .
$$

Then $\eta$ is a continuous deformation from $\left(B_{\rho}, B_{\rho} \backslash\{0\}\right)$ to $\left(B_{\rho} \cap \Phi^{0}, B_{\rho} \cap \Phi^{0} \backslash\{0\}\right)$. By homotopy invariance of a homology group and the contractibility of $B_{\rho} \backslash\{0\}$, we have

$$
C_{q}(\Phi, 0)=H_{q}\left(B_{\rho} \cap \Phi^{0}, B_{\rho} \cap \Phi^{0} \backslash\{0\}\right) \cong H_{q}\left(B_{\rho}, B_{\rho} \backslash\{0\}\right) \cong 0 \quad \text { for all } q \in \mathbb{Z} \text {. }
$$

The proof is complete.

We remark here that in [23] the similar idea for computing the critical groups at 0 was presented for a single elliptic equation. For (GS), the conditions used in [23] can be formulated as

$\left(\tilde{F}_{0}\right)$ there are $\delta>0$ and $1<\sigma<2$ such that

$$
\begin{aligned}
& \text { (i) } \quad F(x, z) \geq c|z|^{\sigma} \quad \text { for } z \in \mathbb{R}^{2} \text { with }|z| \leq \delta, x \in \Omega \text {, } \\
& \text { (ii) } \quad 2 F(x, z)-(\nabla F(x, z), z)>0 \quad \text { for all } z \neq 0, x \in \Omega \text {. }
\end{aligned}
$$

We note here that $\left(\tilde{F}_{0}\right)$ is not comparable with $\left(F_{0}\right)$ since $\left(F_{0}\right)$ is a local condition and although $\left(F_{0}\right)$ implies $\left(\tilde{F}_{0}\right)(\mathrm{i})$ but $\left(\tilde{F}_{0}\right)(\mathrm{ii})$ is a global condition.

Now we verify the compactness for the functional $\Phi$ and compute the critical groups of $\Phi$ at infinity. To do this, we rewrite the functional $\Phi$ as

$$
\Phi(z)=\frac{1}{2} \int_{\Omega}\left(|\nabla z|^{2}-\left(B_{\infty} z, z\right)\right) d x-\int_{\Omega} G(x, z) d x, \quad z \in E
$$

where $2 G(x, z)=2 F(x, z)-\left(B_{\infty} z, z\right)$.

Lemma 3.2 Let $F$ satisfy $\left(F_{\infty}\right)$ and $\left(F_{\infty 1}\right)$.

(i) The functional $\Phi$ is coercive on $E$ and satisfies the Palais-Smale condition.

(ii) $C_{q}(\Phi, \infty) \cong \delta_{q, 0} \mathbb{Z}, q \in \mathbb{Z}$.

Proof (i) First, $\left(F_{\infty}\right)$ implies $(F)$ while $\left(F_{\infty 1}\right)$ implies

$$
\lim _{|z| \rightarrow \infty} G(x, z)=-\infty \quad \text { uniformly in } \Omega
$$

We will prove that

$$
\Phi(z) \rightarrow \infty \quad \text { as }\|z\| \rightarrow \infty, z \in E .
$$

Assume that there is a sequence $\left\{z_{n}\right\} \subset E$ such that for some $M>0$, it holds

$$
\Phi\left(z_{n}\right) \leq M \quad \text { for all } n \in \mathbb{N}
$$




$$
\left\|z_{n}\right\| \rightarrow \infty \quad \text { as } n \rightarrow \infty
$$

Set $\tilde{z}_{n}=\frac{z_{n}}{\left\|z_{n}\right\|}$. Then $\left\|\tilde{z}_{n}\right\|=1$ for all $n \in \mathbb{N}$. Up to a subsequence, we may assume that there is $\tilde{z}_{0} \in E$ such that

$$
\left\{\begin{array}{l}
\tilde{z}_{n} \rightarrow \tilde{z}_{0} \quad \text { weakly in } E, \\
\tilde{z}_{n} \rightarrow \tilde{z}_{0} \quad \text { strongly in } L^{p}(\Omega) \times L^{p}(\Omega), 1 \leq p<2^{*} \\
\tilde{z}_{n}(x) \rightarrow \tilde{z}_{0}(x) \quad \text { for a.e. } x \in \Omega .
\end{array}\right.
$$

By (3.13) one has that for some constant $c_{1}>0$,

$$
G(x, z) \leq c_{1} \quad \text { for } x \in \Omega, z \in \mathbb{R}^{2} .
$$

Therefore by (3.14) we deduce that

$$
\frac{M}{\left\|z_{n}\right\|^{2}} \geq \frac{\Phi\left(z_{n}\right)}{\left\|z_{n}\right\|^{2}} \geq \frac{1}{2}\left\|\tilde{z}_{n}\right\|^{2}-\frac{1}{2} \int_{\Omega}\left(B_{\infty} \tilde{z}_{n}, \tilde{z}_{n}\right) d x-\frac{c_{1}|\Omega|}{\left\|z_{n}\right\|^{2}}
$$

Taking $n \rightarrow \infty$ in (3.18), it follows from (3.15) and (3.16) that

$$
\limsup _{n \rightarrow \infty}\left\|\tilde{z}_{n}\right\|^{2} \leq \int_{\Omega}\left(B_{\infty} \tilde{z}_{0}, \tilde{z}_{0}\right) d x
$$

On the other hand, we have by the lower semi-continuity of the norm that

$$
\int_{\Omega}\left(B_{\infty} \tilde{z}_{0}, \tilde{z}_{0}\right) d x \leq\left\|\tilde{z}_{0}\right\|^{2} \leq \liminf _{n \rightarrow \infty}\left\|\tilde{z}_{n}\right\|^{2}
$$

Thus

$$
\lim _{n \rightarrow \infty}\left\|\tilde{z}_{n}\right\|^{2}=\left\|\tilde{z}_{0}\right\|^{2}
$$

By (3.16), (3.21) we get

$$
\tilde{z}_{n} \rightarrow \tilde{z}_{0} \text { strongly in } E \text { and }\left\|\tilde{z}_{0}\right\|^{2}=\int_{\Omega}\left(B_{\infty} \tilde{z}_{0}, \tilde{z}_{0}\right) d x .
$$

Hence $\left\|\tilde{z}_{0}\right\|=1$ and $\tilde{z}_{0}$ is an eigenvector corresponding to the first eigenvalue $\lambda_{1}\left(B_{\infty}\right)=1$. It follows that $\tilde{z}_{0} \neq 0$ for almost every $x \in \Omega$ and then

$$
\left|z_{n}(x)\right| \rightarrow \infty \quad \text { for a.e. } x \in \Omega \text {. }
$$

Now it follows from (3.13), (3.23) and the Fatou lemma that

$$
\begin{aligned}
M & \geq \frac{1}{2}\left\|z_{n}\right\|^{2}-\frac{1}{2} \int_{\Omega}\left(B_{\infty} z_{n}, z_{n}\right) d x-\int_{\Omega} G\left(x, z_{n}(x)\right) d x \\
& \geq-\int_{\Omega} G\left(x, z_{n}(x)\right) d x \rightarrow \infty \quad \text { as } n \rightarrow \infty .
\end{aligned}
$$

This is a contradiction. Thus $\Phi$ is coercive on $E$. 
By the coercivity of $\Phi$, a Palais-Smale sequence $\left\{z_{n}\right\}$ of $\Phi$ must be bounded. Since $F$ has a subcritical growth, a standard argument shows that $\left\{z_{n}\right\}$ has a convergent subsequence.

(ii) Since $\Phi$ is coercive and weakly lower semi-continuous, $\Phi$ is bounded from below. Take $a<\inf _{z \in E} \Phi(z)$. Then

$$
C_{q}(\Phi, \infty)=H_{q}\left(E, \Phi^{a}\right) \cong H_{q}(E, \emptyset) \cong \delta_{q, 0} \mathbb{Z}, \quad q \in \mathbb{Z}
$$

The proof is complete.

Lemma 3.3 Let F satisfy $\left(F_{\infty}\right)$ and $\left(F_{\infty 2}^{ \pm}\right)$.

(i) $\Phi$ satisfies the Cerami condition.

(ii) $C_{q}(\Phi, \infty) \cong \delta_{q, m_{\infty+n_{\infty}}} \mathbb{Z}$ if $\left(F_{\infty 2}^{+}\right)$holds.

(iii) $C_{q}(\Phi, \infty) \cong \delta_{q, m_{\infty}} \mathbb{Z}$ if $\left(F_{\infty 2}^{-}\right)$holds.

Proof (i) Let $\left\{z_{n}\right\} \subset E$ be such that

$$
\left(1+\left\|z_{n}\right\|\right)\left\|\Phi^{\prime}\left(z_{n}\right)\right\| \rightarrow 0 \quad \text { as } n \rightarrow \infty .
$$

We only need to show that $\left\{z_{n}\right\}$ is bounded in $E$. Suppose, by the way of contradiction, that

$$
\left\|z_{n}\right\| \rightarrow \infty \quad \text { as } n \rightarrow \infty
$$

Denote $\bar{z}_{n}=\frac{z_{n}}{\left\|z_{n}\right\|}$, then $\left\|\bar{z}_{n}\right\|=1$. Passing to a subsequence if necessary, we may assume that there is $\bar{z} \in E$ such that as $n \rightarrow \infty$,

$$
\begin{cases}\bar{z}_{n} \rightarrow \bar{z} & \text { strongly in } L^{2}(\Omega) \times L^{2}(\Omega), \\ \bar{z}_{n} \rightarrow \bar{z} & \text { weakly in } E, \\ \bar{z}_{n}(x) \rightarrow \bar{z}(x) & \text { almost everywhere in } \Omega\end{cases}
$$

By $\left(F_{\infty}\right)$ and (3.26) we deduce that $\left\{\frac{\nabla F\left(x, z_{n}(x)\right)-B_{\infty}(x) z_{n}(x)}{\left\|z_{n}\right\|}\right\}$ is bounded in $L^{2}(\Omega) \times L^{2}(\Omega)$ and

$$
\frac{\nabla F\left(x, z_{n}\right)-B_{\infty}(x) z_{n}}{\left\|z_{n}\right\|} \rightarrow 0 \quad \text { almost everywhere in } \Omega .
$$

Therefore

$$
\frac{\nabla F\left(x, z_{n}\right)-B_{\infty}(x) z_{n}}{\left\|z_{n}\right\|} \rightarrow 0 \quad \text { in } L^{2}(\Omega) \times L^{2}(\Omega) .
$$

For $w \in E$, we have

$$
\begin{aligned}
\frac{\left\langle\Phi^{\prime}\left(z_{n}\right), w\right\rangle}{\left\|z_{n}\right\|}= & \int_{\Omega} \nabla \bar{z}_{n} \nabla w-\left(B_{\infty}(x) \bar{z}_{n}, w\right) d x \\
& -\int_{\Omega}\left(\frac{\nabla F\left(x, z_{n}\right)-B_{\infty}(x) z_{n}}{\left\|z_{n}\right\|}, w\right) d x .
\end{aligned}
$$

Letting $n \rightarrow \infty$, it follows that

$$
\int_{\Omega} \nabla \bar{z} \nabla w-\left(B_{\infty}(x) \bar{z}, w\right) d x=0 \quad \text { for } w \in E
$$


Taking $w=\bar{z}_{n}$ in (3.29) and using (3.25), (3.28), we obtain that

$$
1=\int_{\Omega}\left(B_{\infty}(x) \bar{z}, \bar{z}\right) d x
$$

Taking $w=\bar{z}$ in (3.30), we obtain that

$$
\|\bar{z}\|^{2}=\int_{\Omega}\left(B_{\infty}(x) \bar{z}, \bar{z}\right) d x=1
$$

Therefore $\left\|\bar{z}_{n}\right\| \rightarrow\|\bar{z}\|=1$ and thus

$$
\bar{z}_{n} \rightarrow \bar{z} \quad \text { strongly in } E
$$

and $\bar{z}$ is an eigenvector of $\left(\mathrm{L}_{B_{\infty}}\right)$ associated with eigenvalue 1. It follows that $\bar{z} \in E_{\infty}$. Write $z_{n}=y_{n}+w_{n}$, where $y_{n} \in E_{\infty}, w_{n} \in E_{\infty}^{\perp}$, then

$$
\frac{\left\|y_{n}\right\|}{\left\|z_{n}\right\|} \rightarrow 1 \quad \text { as } n \rightarrow \infty
$$

By $\left(F_{\infty 2}^{ \pm}\right)$there exist $\delta>0$ and $N \in \mathbb{N}$ such that

$$
\left|\left\langle\Phi^{\prime}\left(z_{n}\right), y_{n}\right\rangle\right|=\left| \pm \int_{\Omega}\left(\nabla F\left(x, z_{n}\right)-B_{\infty}(x) z_{n}, y_{n}\right) d x\right| \geq \delta \quad \text { for all } n \geq N .
$$

This implies that

$$
\left\|\Phi^{\prime}\left(z_{n}\right)\right\|\left\|z_{n}\right\| \geq \delta \quad \text { for all } n \geq N
$$

This is a contradiction with (3.25).

(ii) We apply Proposition 2.2. Set $\mathcal{L}:=I-T_{B_{\infty}}$ and

$$
\Psi(z)=\frac{1}{2} \int_{\Omega}\left(\left(B_{\infty}(x) z, z\right)-2 F(x, z)\right) d x .
$$

Then $\Phi$ can be rewritten as

$$
\Phi(z)=\frac{1}{2}\langle\mathcal{L} z, z\rangle+\Psi(z), \quad z \in E .
$$

By Lemma 3.1, $\Phi$ satisfies the Cerami condition and hence possesses the deformation property. From $\left(F_{\infty}\right)$ one sees that $\Psi^{\prime}$ satisfies

$$
\Psi^{\prime}(z)=o(\|z\|), \quad\|z\| \rightarrow \infty .
$$

Now we show that $\Phi$ satisfies the angle condition $\left(\mathcal{A C}_{\infty}^{-}\right)$at infinity with respect to the orthogonal decomposition $E=E_{\infty} \oplus E_{\infty}^{\perp}$ when $\left(F_{\infty 2}^{+}\right)$holds. Suppose it is not true, then for each $n \in \mathbb{N}$, there are $z_{n} \in E, z_{n}=y_{n}+w_{n}, y_{n} \in E_{\infty}, w_{n} \in E_{\infty}^{\perp}$ such that

$$
\left\|z_{n}\right\| \geq n, \quad\left\|w_{n}\right\| \leq \frac{1}{n}\left\|z_{n}\right\| \quad \text { for all } n \in \mathbb{N},
$$


and

$$
\left\langle\Phi^{\prime}\left(z_{n}\right), y_{n}\right\rangle>0 \quad \text { for all } n \in \mathbb{N} \text {. }
$$

It follows from (3.35) that

$$
\left\|z_{n}\right\| \rightarrow \infty, \quad \frac{\left\|y_{n}\right\|}{\left\|z_{n}\right\|} \rightarrow 1, \quad n \rightarrow \infty
$$

By $\left(F_{\infty 2}^{+}\right)$we have that for $N \in \mathbb{N}$,

$$
\left\langle\Phi^{\prime}\left(z_{n}\right), y_{n}\right\rangle=\left\langle\Psi^{\prime}\left(z_{n}\right), y_{n}\right\rangle=-\int_{\Omega}\left(\nabla F\left(x, z_{n}\right)-B_{\infty}(x) z_{n}, y_{n}\right) d x \leq-\delta, \quad n \geq N
$$

this contradicts (3.36). Therefore $\left(\mathcal{A C}_{\infty}^{-}\right)$holds, and by Proposition 2.2 we have

$$
C_{q}(\Phi, \infty)=\delta_{q, m_{\infty}+n_{\infty}} \mathbb{Z}, \quad q \in \mathbb{Z}
$$

(iii) This case is proved in a similar way.

The proof is finished.

\section{Proofs of main theorems}

In this section we give the proofs of main theorems in this paper.

Proof of Theorem 1.1 By $\left(F_{\infty}\right)$, the functional $\Phi$ takes the form

$$
\Phi(z)=\frac{1}{2}\langle\mathcal{L} z, z\rangle+\Psi(z), \quad z \in E
$$

where $\mathcal{L}=I-T_{B_{\infty}}$ is a bounded self-adjoint linear operator, $\Psi \in C^{1}(E, \mathbb{R})$ with a compact gradient $\Psi^{\prime}$ satisfying

$$
\Psi^{\prime}(z)=o(\|z\|), \quad\|z\| \rightarrow \infty
$$

Since $n_{\infty}=0$, the problem is no resonance at infinity and thus $\Psi$ satisfies the Palais-Smale condition. By Proposition 2.2(1) we have

$$
C_{q}(\Phi, \infty) \cong \delta_{q, m_{\infty}} \mathbb{Z}, \quad q \in \mathbb{Z}
$$

Therefore $\Phi$ has a critical point $z_{*}$ satisfying

$$
C_{m_{\infty}}\left(\Phi, z_{*}\right) \not 0 .
$$

By Lemma 3.1 we have

$$
C_{q}(\Phi, 0) \cong 0, \quad \forall q \in \mathbb{Z}
$$

By (4.2) and (4.3), we see that $z_{*} \neq 0$ and then is a nontrivial weak solution of (GS). 
Proof of Theorem 1.2 By $\left(F_{\infty}\right),\left(F_{\infty 1}\right)$ and Lemma 3.2, we have

$$
C_{q}(\Phi, \infty) \cong \delta_{q, 0} \mathbb{Z}, \quad q \in \mathbb{Z}
$$

Therefore $\Phi$ has a critical point $z_{*}$ satisfying

$$
C_{0}\left(\Phi, z_{*}\right) \supsetneqq 0
$$

We still have (4.3). Thus $z_{*} \neq 0$ is a nontrivial weak solution of (GS). In fact, $z^{*}$ is a global minimizer of $\Phi$.

Assume that $F(x, z)$ is even in $z$. We will employ Proposition 2.3 to prove the multiplicity in Theorem 1.2. Now $\Phi$ is even, $\Phi(0)=0$. By Lemma 3.2, $\Phi$ satisfies the Palais-Smale condition and is bounded from below following from the coercivity.

We verify (2.5). Let $E_{k}$ be a $k$-dimensional subspace of $E$. For $z \in E_{k}$, as arguments in the proof of Lemma 3.1, we have

$$
\Phi(z) \leq \frac{1}{2}\|z\|^{2}-c_{2}\|z\|_{L^{\sigma}}^{\sigma}+c_{3}\|z\|_{L^{p}}^{p} .
$$

Since $\sigma<2<p$ and all norms on $E_{k}$ are equivalent, we get that for $\rho_{k}>0$ small enough,

$$
\sup _{z \in X_{k} \cap S_{\rho_{k}}} \Phi(z)<0 .
$$

With all the conditions of Proposition 2.3 being verified, we get the conclusion that $\Phi$ has a sequence of critical values $c_{k}<0$ satisfying $c_{k} \rightarrow 0$ as $k \rightarrow \infty$. Thus (GS) has infinitely many nontrivial weak solutions in $E$. The proof is finished.

Proof of Theorem 1.3 By a similar argument, it follows from Lemma 3.1 and Lemma 3.3.

We conclude the paper with further comments and remarks.

Remark 4.1 (i) In Theorem 1.1, when $\lambda_{1}\left(B_{\infty}\right)>1$ which implies $m_{\infty}=0$ and $F$ is even in $z$, by the same arguments as the last part of the proof of Theorem 1.2, one can show that (GS) has infinitely many nontrivial weak solutions in $E$ with negative energies which converge to zero.

(ii) In Theorem 1.2, one nontrivial solution could be obtained if $\left(F_{\infty 1}\right)$ is replaced by the nonquadraticity condition [24]

$$
\lim _{|z| \rightarrow \infty}\{(\nabla F(x, z), z)-2 F(x, z)\}=\infty \quad \text { uniformly for a.e. } x \in \Omega . \quad\left(\tilde{F}_{\infty 1}^{+}\right)
$$

Indeed, $\left(\tilde{F}_{\infty 1}^{+}\right)$is equivalent to

$$
\lim _{|z| \rightarrow \infty}\{(\nabla G(x, z), z)-2 G(x, z)\}=\infty \quad \text { uniformly for a.e. } x \in \Omega, \quad\left(\tilde{G}_{\infty 1}\right)
$$

which implies $\left(F_{\infty 1}\right)$, i.e.,

$$
G(x, z) \rightarrow-\infty \quad \text { uniformly for a.e. } x \in \Omega \text {. }
$$

$\left(F_{\infty 1}\right)$ is weaker than $\left(\tilde{F}_{\infty 1}\right)$. 
(iii) The result for one nontrivial solution in Theorem 1.2 is valid when $F$ satisfies $\left(F_{0}\right)$, $\left(F_{\infty}\right)$ and the nonquadraticity condition [24]

$$
\lim _{|z| \rightarrow \infty}\{(\nabla F(x, z), z)-2 F(x, z)\}=-\infty \quad \text { uniformly for a.e. } x \in \Omega . \quad\left(\tilde{F}_{\infty 1}^{-}\right)
$$

Indeed, in this case, $\Phi$ satisfies the Cerami condition and $\Phi$ has a saddle point structure at infinity with respect to $E=E_{\infty} \oplus E_{\infty}^{\perp}$ in the sense that $\Phi$ is bounded from below on $E_{\infty}^{\perp}$ and is anti-coercive on $E_{\infty}$. Then Proposition 3.8 in [18] is applied to get $C_{1}(\Phi, \infty) \nsucceq 0$.

(iv) In Theorem 1.3, the global condition $\left(F_{\infty}^{ \pm}\right)$is somewhat abstract and has been used in [12]. It could be verified if $\pm\left(\nabla F(x, z)-B_{\infty}(x) z, z\right)$ acts as $|z|^{s+1}$ near infinity for any $s \in(0,1)$ (see $[20,25])$. See [12] for more comparisons.

Remark 4.2 In Theorem 1.2, we proved the multiplicity result by a critical point theorem in [22] when $\Phi$ is even. This result is completely new for gradient systems. Since the critical groups of $\Phi$ at both zero and infinity are clearly computed, when $\Phi$ is even, the Morse equality may provide us an idea to give a different proof provided we have in hand the following basic conclusion.

$(\star)$ If $z^{*}$ is a solution of (HS), then $C_{q}\left(\Phi, z^{*}\right) \neq 0$ for finitely many $q \in \mathbb{N}$.

Let $(\star)$ hold and let $F$ be even. We prove the multiplicity for (GS) in Theorem 1.2 via Morse theory. Assume that (GS) has only finitely many pairs of nontrivial solutions. Denote $\mathcal{K}=\left\{0, \pm z_{1}, \pm z_{2}, \ldots, \pm z_{k}\right\}$. Then by the Morse equality, one has that

$$
\sum_{q=0}^{\infty}(-1)^{q} \sum_{z \in \mathcal{K}} \operatorname{dim} C_{q}(\Phi, z)=\sum_{q=0}^{\infty}(-1)^{q} \operatorname{dim} C_{q}(\Phi, \infty)
$$

By $(\star),(4.3)$ and $(4.4)$, it follows that

$$
\sum_{q=0}^{\infty}(-1)^{q} 2 \sum_{i=1}^{k} \operatorname{dim} C_{q}\left(\Phi, z_{i}\right)=1
$$

a contradiction. Similarly, if $(\star)$ is valid and $F$ is even, then we have the same multiplicity result in Theorems 1.1 and 1.3.

We note here that the conclusion $(\star)$ is valid for $\Phi$ is of $C^{2}$. A natural problem arises here whether or not that $(\star)$ is valid for a $C^{1}$ functional. It is still open to the best of our knowledge. We will focus on this problem in near future.

Competing interests

The authors declare that they have no competing interests.

Authors' contributions

Both authors contributed equally to the manuscript and read and approved the final manuscript.

Acknowledgements

Supported by NSFC11271264, NSFC11171204 and PHR201106118. 


\section{References}

1. Rabinowitz, P: Minimax Methods in Critical Point Theory with Application to Differential Equations. CBMS, vol. 65. Am Math. Soc., Providence (1986)

2. Chang, KC: An extension of the Hess-Kato theorem to elliptic systems and its applications to multiple solutions problems. Acta Math. Sin. 15, 439-454 (1999)

3. Chang, KC: Principal eigenvalue for weight in elliptic systems. Nonlinear Anal. 46, 419-433 (2001)

4. Landesman, E, Lazer, A: Nonlinear perturbations of linear eigenvalues problem at resonance. J. Math. Mech. 19 609-623 (1970)

5. Bartsch, T, Chang, KC, Wang, Z-Q: On the Morse indices of sign changing solution of nonlinear elliptic problem. Math. Z. 233, 655-677 (2000)

6. Liu, JQ, Wu, SP: A note on a class of sublinear elliptic equation. Research Report 84, Peking University (1997)

7. da Silva, ED: Multiplicity of solutions for gradient systems. Electron. J. Differ. Equ. 2010, 64 (2010)

8. da Silva, ED: Multiplicity of solutions for gradient systems using Landesman-Lazer conditions. Abstr. Appl. Anal. 2010, Article ID 237826 (2010)

9. Furtado, MF, de Paiva, FOV: Multiplicity of solutions for resonant elliptic systems. J. Math. Anal. Appl. 319, 435-449 (2006)

10. Furtado, MF, de Paiva, FOV: Multiple solutions for resonant elliptic systems via reduction method. Bull. Aust. Math. Soc. 82, 211-220 (2010)

11. Li, C, Liu, S: Homology of saddle point reduction and applications to resonant elliptic systems. Nonlinear Anal. 81 236-246 (2013)

12. Lü, L, Su, J: Solutions to a gradient system with resonance at both zero and infinity. Nonlinear Anal. 74, 5340-5351 (2011)

13. Liu, JQ, Su, J: Remarks on multiple nontrivial solutions for quasi-linear resonant problems. J. Math. Anal. Appl. 258 209-222 (2001)

14. Chang, KC: Infinite Dimensional Morse Theory and Multiple Solutions Problems. Birkhäuser, Boston (1993)

15. Mawhin, J, Willem, M: Critical Point Theory and Hamiltonian Systems. Springer, Berlin (1989)

16. Bartolo, P, Benci, V, Fortunato, D: Abstract critical point theorems and applications to nonlinear problems with 'strong' resonance at infinity. Nonlinear Anal. 7, 981-1012 (1983)

17. Cerami, G, Un criterio di esistenza per i punti critici su varietà illimitate. Rend. - Ist. Lomb., Accad. Sci. Lett., a Sci. Mat. Fis. Chim. Geol. 112, 332-336 (1978)

18. Bartsch, T, Li, S: Critical point theory for asymptotically quadratic functionals and applications to problems with resonance. Nonlinear Anal. 28, 419-441 (1997)

19. Wang, Z-Q: Multiple solutions for indefinite functionals and applications to asymptotically linear problems. Acta Math. Sin. New Ser. 5(2), 101-113 (1989)

20. Su, J: Nontrivial periodic solutions for the asymptotically linear Hamiltonian systems with resonance at infinity. J. Differ. Equ. 145(2), 252-273 (1998)

21. Su, J, Zhao, L: An elliptic resonance problem with multiple solutions. J. Math. Anal. Appl. 319, 604-616 (2006)

22. Wang, Z-Q: Nonlinear boundary value problems with concave nonlinearities near the origin. Nonlinear Differ. Equ. Appl. 8, 15-33 (2001)

23. Moroz, V: Solutions of superlinear at zero elliptic equations via Morse theory. Topol. Methods Nonlinear Anal. 10 387-397 (1997)

24. Costa, DG, Magalhães, CA: Variational elliptic problems which are nonquadratic at infinity. Nonlinear Anal. 23 , 1401-1412 (1994)

25. Su, J, Tang, C: Multiplicity results for semilinear elliptic equations with resonance at higher eigenvalues. Nonlinear Anal. 44, 311-321 (2001)

\section{Submit your manuscript to a SpringerOpen ${ }^{\circ}$ journal and benefit from:}

- Convenient online submission

Rigorous peer review

- Immediate publication on acceptance

- Open access: articles freely available online

- High visibility within the field

- Retaining the copyright to your article 